\title{
EXPLORING THE EFFECTIVENESS OF ONLINE Role-PLAy GaMing IN THE ACQUiSition OF COMPLEX AND TACIT KNOWLEDGE
}

\author{
Christian Wagner, City University of Hong Kong, c.wagner@cityu.edu.hk \\ Victor Dibia, City University of Hong Kong, dibia.victor@my.cityu.edu.hk
}

\begin{abstract}
We report the findings from an experiment concerning the impact of gaming on the acquisition of simple vs. complex knowledge, as well as tacit knowledge. An experimental study involving 35 novice World of Warcraft gamers shows that gaming leads to knowledge acquisition, which is higher for fact-based knowledge than for complex, planningtype knowledge. The results also indicate that subjects acquire more knowledge than they are able to recall. Results concerning the impact of chauffeuring, i.e., game demonstration by an experimenter prior to subjects' own task work are inconclusive, but hint at the value of chauffeuring to flatten the learning curve.
\end{abstract}

Keywords: Knowledge management, computer games and tacit knowledge

\section{INTRODUCTION}

Computer games are argued to be effective tools for acquiring knowledge, due to their implementation of the entire learning process of practice, feedback for reinforcement, and rewards for behavior improvement $[12,7,11,22]$. Whereas some game forms may promote relatively little knowledge transfer, i.e., "shooter" games, online role playing applications require players to identify with their role, grasp role-specific complex concepts (e.g., attributes of the player's game character), and formulate longer term plans to achieve objectives. Further, they embed the required learning in captivating scenarios that increase motivation, while accommodating multiple learning styles and skills [10]. Brown and Douglas [6] argue that the skill accumulation in World of Warcraft was transferable and should be considered a positive factor in hiring decisions. This raises the additional question of how capable games are in enabling tacit knowledge transfer. After all, Smith [23] argues that tacit knowledge is one of the key skills required in the work place and that "organizations must begin to create worker-centered environments to encourage the open sharing and use of all forms of knowledge". Tacit knowledge, by definition, is a form of knowledge that cannot be transferred simply by verbalization.

The transfer of tacit knowledge in particular, is difficult, because by definition, it is not explicated, i.e., it cannot be told. The learner has to develop a knowledge representation that matches the original, based on a process that may include (re-)creating experiences which lead to the knowledge development. What makes this process even more challenging is the fact that the learner also cannot tell whether he or she has mastered the knowledge, again, because it cannot be explicated, but has to be demonstrated indirectly. Consequently, the possible demonstration of tacit knowledge acquisition by learners would be a significant achievement, since otherwise, tacit knowledge transfer is recognized as a highly effortful process, relying on resource intensive processes such as apprenticeship learning [17]. Our research is thus intended to explore the question of knowledge acquisition, both complex and tacit, through gaming. Using the popular online game World of Warcraft as our test bed, we experimentally sought to explore the effects of knowledge complexity on the process of knowledge transfer within games, and the scenario where people acquire some form of knowledge but cannot tell.

At the same time, we realized that while online role-playing games appear to have significant potential to facilitate knowledge transfer, they are not without challenges. Specifically, in recent years, games have been reported to exhibit a steep learning curve, which might hamper the knowledge transfer, as game players may fail and give up. Thus we also intended to show whether complex tasks benefit from a period of supervision ("chauffeured" gaming) to produce more successful learning outcomes.

The remainder of the article is organized as follows. First we provide a brief background on role-playing games, virtual worlds and knowledge complexity. Next we present our research model and experimental setup. Then we 


\section{Issues in Information Systems}

Volume 14, Issue 2, pp.367-374, 2013

introduce preliminary results, followed by a discussion of our preliminary findings. We close with an interpretation of our results, limitations, and concluding remarks.

\section{RESEARCH BACKGROUND}

\section{Role Playing Games (RPGs) and Virtual Worlds}

Virtual worlds are frequently described as massive multiplayer online environments, represented as highly immersive games or "game-like" scenarios where "residents" can engage in socialization, entertainment, education, and commerce [2]. Virtual worlds emerged in the 1980s, not long after the first multi-user time-sharing computer systems. At that time, however, users interacted with the environment through terse, text-based exchanges. The evolution of high-speed data connections and high resolution, fast graphics have taken interactivity and thus immersion to new levels, enabling for instance Lindenlab's Second life to render a virtual world to users in real time. The evolutionary nature of virtual worlds also led to an evolution in the definition of the term. Wu \& Liu [25] for instance stressed the interactivity of virtual worlds, whereas [5] defined a virtual world as a "computer-based, simulated multi-media environment, usually running over the Web, and designed so that users can 'inhabit' and interact via their own graphical self-representations known as avatars" (p. 233). Others $[1,3]$ have stressed the importance of the persistence of the virtual world, i.e., the fact that it exists and evolves even when participants $\log$ off. Considering all these definitions together, we describe virtual worlds as computer based environments involving a large number of players, operating in a persistent and immersive online environment, typically in 3D (or simulated 3D).

For the purpose of this article, however, we are interested in a subset of virtual worlds, namely games, which are "purposeful" virtual worlds. By purposeful, we mean that users have one or more objectives (e.g., "slay the dragon"), receive pay-offs for the achievement of objectives, operate in a structured environment (e.g., with physical structures such as buildings), are subject to rules (e.g., rule of gravity), and interact with other players or with the game environment. Most important among these is the objective and reward system. Whereas there are no predefined objectives for instance in Second Life, and thus players need define for themselves why they participate, a game has clear goals and pay-offs for achieving them. Goals are typically presented in hierarchical order, from minor goals (e.g., finding a resource) to quest goals (completing a set of tasks which together form a "quest"), to high-level goals that lead from "leveling up" to "winning the game" (if possible). Such a goal and pay-off system is closely associated with learning, as learning requires reinforcement, and thus the ability to reward achievement of objectives. It is frequently believed that the clever design of games with many goals and corresponding rewards account for the level of learning that takes place within them [8]. Role Playing Games (RPGs) are a subset of online games that people typically participate in which diverse motivations - achievement, social and immersion [27]. These games are oftentimes played by millions of people simultaneously, and thus considered "massively multiplayer online role-playing games" (MMORPG). Participants of MMORPG's are on the average, 26 years old and spend 22 hours each week in within the games [26]. This high popularity makes them an ideal experimental environment to explore knowledge acquisition within the gaming context.

\section{Role of Immersion}

Several factors make Virtual Worlds particularly amenable to the process of learning. One of such factors is immersion. Immersion refers to the feeling of being present within a virtual space and identifying one's character as oneself [24]. This feeling of embodiment within virtual worlds as a communication medium provides a richer layer, which is capable of capturing and representing more tacit forms of human interactions [21]. Elsewhere, immersion in a virtual reality environment has also been demonstrated to improve the creation of problem related knowledge structures [9].

\section{World of Warcraft}

World of Warcraft (or WoW), a MMORPG, is one of the most successful online games of all times in terms of number of users, number of same-time users, revenue generated for the company, and size of the in-game economy, i.e., the US Dollar equivalent of the goods bought and sold within the game or at its fringes. WoW creates a fantasy environment where players play against the environment $(\mathrm{PvE})$ or against other players $(\mathrm{PvP})$ to capture resources 


\section{Issues in Information Systems \\ Volume 14, Issue 2, pp.367-374, 2013}

and gain experience that help them ascend to increasingly higher levels of capabilities and reputation. The game is situated in the fictitious Azeroth, where two factions, the Horde and Alliance fight each other (PvP), but where players also frequently are tasked to cleanse a diseased environment (PvE). Players can take on basic tasks, such as mining for ores, or killing beasts, but achieve higher pay-offs in form of currency or experience points by taking on more complex quests. Leveling up, the rise in capabilities, depends on experience points (XPs), and is highly nonlinear. To advance from Level 1 to Level 60, for instance, requires 3.38 Million XPs, yet moving from level 60 to 70 needs 4.77 MXPs, and from 80 to 85 takes 22.18 MXPs [25]. To achieve these ever larger point numbers, players must engage in more complex quests with heavier weaponry, and possibly with the help of other players.

Typical gaming-oriented virtual worlds such as WoW are not easily adapted to educational purposes because of their pre-defined structure and reward system, as well as the fantasy environment [14]. While some proponents stress the ability to learn transferable skills such as team work coordination, the purpose of this research is domain specific knowledge acquisition. Thus, WoW was supposed to serve as proof of concept that a MMORPG could effectively transfer knowledge. The expectation would then be that real-world uses elsewhere, where domain knowledge transfer was required, would make use of a similar game engine, but with domain specific content (e.g., SimuLearn).

\section{RESEARCH DESIGN}

To examine the acquisition of complex and tacit knowledge, we carried out an experiment that would explore the impact of knowledge complexity, knowledge tacitness, and the impact of chauffeured learning. Each of these dimensions and their role in the research design is outlined below.

\section{Knowledge Complexity}

Online games have been shown to possess the ability to transfer knowledge and support learning $[12,7,11,22]$. However, it is well understood that there are different levels of learning, with higher levels being more difficult to attain, as for instance represented in Bloom's taxonomy of learning [4]. Bloom differentiates levels from a basic level of fact remembering, to mid-levels of application, to the highest level, of creativity. Another approach is more task specific and considers knowledge as a requirement for problem solving. Within a problem solving paradigm [e.g., 20,15], subjects have to learn to move from a less desirable to a more-desirable state. To do so, they have to understand and learn factual knowledge about physical objects and non-physical concepts. They also have to understand the connection between causes and effects (i.e., means-ends learning [16]), and need to learn and develop plans (mapping out multiple steps to achieve a goal, based on cause-effectrelationships).

Bloom's taxonomy and the problem solving paradigm are complementary in that they both recognize the need for (a) fact knowledge, such as the description of physical objects and attributes; (b) concept and cause-effect knowledge that arises from analysis and can be applied in task completion, and (c) plan knowledge that requires the understanding of multiple cause-effect steps, and the need to combine components in a process of synthesis.

In this experimental study, we identified several further knowledge sub-types: Physical Object (PO) questions asked for physical items, such as "what are first creatures you meet on your first quest?" We expected these to be most easy to answer, since they required only factual information, which was easily observable. Physical Object Attribute (POA) questions referred to attributes of physical objects. An example would be "what is the most typical item dropped by the ... [creature]?" Again, we expected these to be easily answerable, since they asked for factual, clearly observable status information. Concept (CON) questions were next in our set of questions. These questions also related to status information, but about non-physical, and thus more difficult to investigate objects (which are also less amenable to multi-media representation). An example would be "why stop killing beasts after completing the number required for quest completion? "Cause-effect (CE) questions where variations of the question, "what happens if... " or "how do you achieve effect..." For example, one question was "how do you wield your weapon?" a question whose answer could only be determined through experimentation (trail-and-error). We considered causeeffect questions as more difficult, because they required the understanding of a working principle, and possibly hypothesis testing. Plan (PLAN) questions required understanding of multiple cause-effect steps which, linked together, would lead to achievement of a goal. Frequently they were asked in the form "How do you complete the XYZ quest? Describe the complete procedure." Such questions were more difficult than CE, because they required memorization of a group of related CE links. 
For experimental purposes, we differentiated among the different types in questioning to ensure a broad spectrum of questions, but combined CON, CE, and PLAN into a category complex knowledge (CK) and PO and POA into simple knowledge (SK) in light of the research objective. Our expectation, was that,

H1: Subjects will demonstrate higher ability to recall simple knowledge than complex knowledge.

\section{Explicit vs. Tacit Knowledge}

Tacit knowledge) is a form of knowledge that cannot be easily verbalized or otherwise explicated, as reflected in the statement "knowing more than one can tell". Contrary to explicit knowledge, tacit knowledge is not amenable to the conventional means of knowledge transfer such as verbalizing. This distinction between tacit and explicit knowledge was first identified by Michael Polanyi and discussed in detail in The Tacit Dimension [19]. The formalization and management of tacit knowledge has since been identified as generating inter-organizational competitive advantage [17] and being a key driver for innovation in organizations [13].

Considering the prior task categories, we viewed simple knowledge as highly explicit, easy to observe and easy to report in a questionnaire. If subjects seemingly had attained the knowledge, as demonstrated by their task completion, they should also be able to explicate it. For example, if a subject needed to walk up three flights of stairs, or pick a green object, they should also have awareness of the object color, or number of flights of stairs, and thus be able to report them. We did not expect the same to be true for complex knowledge, such as plans the subjects had to make to achieve a task, or cause-effect links they had to create. We thus hypothesized,

H2a: Subjects will show complete ability to recall simple task related knowledge, following task completion (thus suggesting NO tacit knowledge transfer).

H2b: Subjects will show less than perfect ability to recall complex task related knowledge, following task completion (thus suggesting some tacit knowledge transfer).

\section{Learning-by-doing}

As knowledge acquisition through games can be difficult when the learning curve is steep, we also sought to determine whether chauffeuring subjects would have an impact on knowledge acquisition. We expected that chauffeuring would allow subjects to overcome start-up problems and speed-up their knowledge acquisition effort. We thus hypothesized,

H3a: Subjects who experience chauffeured gaming will demonstrate higher performance in task completion.

H3b: Subjects who experience chauffeured gaming will demonstrate higher performance in reporting acquired knowledge.

\section{Experimental Design}

35 subjects, recruited through advertising and word-of-mouth, participated in the experiment. Subjects, all students at a major university, were rewarded for their participation through cash coupons worth US\$7 each. Subjects were informed of normal experimental procedures, including preservation of their anonymity in data reporting, and their right to withdraw at any time. All subjects were (self-declared) novices in the use of WoW. Approximately $1 / 3$ were women, $2 / 3$ men. Subjects were randomly allocated to treatment groups, as shown in Table 1.

Table 1. Task Groups and Treatment

\begin{tabular}{|l|l|l|l|l|}
\hline & n & Task 1 & Task 2 & Post-Experiment \\
\cline { 1 - 3 } Group 1 & 15 & chauffeured & \multirow{2}{*}{ self-managed } & Questionnaire \\
\hline Group 2 & 20 & self-managed & \\
\hline
\end{tabular}

The overall logic of the experiment required subjects to complete tasks within World of Warcraft, followed by answering skill questions about the tasks and the game in questionnaire format. Groups 1 and 2 differed in that the first group carried out the initial task "chauffeured", watching an experimenter completing the exercise. During the chauffeured portion, the experimenter would complete Task 1, while voicing out actions such as "I am pressing the 


\section{Issues in Information Systems \\ Volume 14, Issue 2, pp.367-374, 2013}

left mouse button", so as to express the factual experience in words, but without explaining deeper aspects of the game, thus not revealing any significant knowledge. Thereafter, subjects would complete Task 1 themselves.

All subjects were asked to complete the same tasks (Task 1 and Task 2). For Task 1, subjects assumed a Level 1 character and carried out quests at that level. Task 2 also required questing, but at a higher difficulty level (more complex quest). Subjects were closely monitored by experimenters, who measured the time taken to complete parts of the exercise, such as returning from a task back to their home base. Post-experiment questions were mostly multiple choice and of the following nature: "How did you interact with a Quest-Giver Character to accept or complete a quest? (Select all that apply) [Answer options: By approaching it|By clicking on it|By running beside it|By jumping through it]". For multiple choice questions, a score of 1 was given if the correct options were chosen and 0.5 if some correct answers were chosen. 0.5 was also given if a mix of correct and wrong options was chosen. Other answers were simply marked 1.0 (right) or 0.0 (wrong).

\section{DATA COLLECTION AND FINDINGS}

35 subjects agreed to participate in the 1-hour long experiment. 27 were able to complete the required two tasks in the allotted time. These 27 subjects also completed the Post-experiment questionnaire, answering 14 task knowledge related questions in all.

Results concerning H1 are shown in Table 2. The table breaks down mean recall values by simple vs. complex knowledge, and based on chauffeured or self-managed completion of the first part of the experiment. To compute significance, both a standard t-test (all subjects) and an unpaired t-test (chauffeured vs. self-managed) were used.

Table 2. T-test of Knowledge Recall Differences

\begin{tabular}{|c|c|c|c|c|c|}
\hline & & SK & CK & $t$ & $\mathbf{p}$ \\
\hline \multirow[t]{3}{*}{ All Subjects } & Mean & 0.8086 & 0.5571 & \multirow[t]{3}{*}{5.196} & \multirow[t]{3}{*}{.000} \\
\hline & Std.Dev. & 0.1792 & 0.1764 & & \\
\hline & $\mathrm{n}$ & $27(35)$ & $27(35)$ & & \\
\hline \multirow[t]{3}{*}{ Chauffeured } & Mean & 0.8413 & 0.5000 & \multirow[t]{3}{*}{5.531} & \multirow[t]{3}{*}{.000} \\
\hline & Std.Dev. & 0.1475 & 0.1775 & & \\
\hline & $\mathrm{n}$ & $14(15)$ & $14(15)$ & & \\
\hline \multirow[t]{3}{*}{ Self-managed } & Mean & 0.7735 & 0.6186 & \multirow[t]{3}{*}{2.128} & \multirow[t]{3}{*}{.027} \\
\hline & Std.Dev. & 0.2084 & 0.1594 & & \\
\hline & $\mathrm{n}$ & $13(20)$ & $13(20)$ & & \\
\hline
\end{tabular}

All differences in recall of simple vs. complex knowledge were significant, with knowledge recall after selfmanaged gaming showing the least difference between simple and complex knowledge.

For the test of H2, we completed another set of t-tests of the recall rates for SK and CK against perfect recall (1.0). The respective results were $t=5.547, p=0.000$ for SK and $t=13.042, p=0.000$ for $\mathrm{CK}$, with $\mathrm{df}=26$ for both.

For the test of $\mathrm{H} 3$, we compared completion rates of the task in chauffeured vs. self-managed mode, using a $\chi^{2}$ test. Of the 8 subjects unable to complete the task, 7 had operated in self-managed mode. According to the participation rates of 15 and 20 subjects in the corresponding cells, we computed $\chi^{2}=3.014, p=0.083(\mathrm{df}=1)$. We further compared average recall rates for all knowledge items in chauffeured vs. self-managed mode, using a t-test. With average overall recall for knowledge in chauffeured mode being 0.6811 vs. 0.6923 in self-managed mode, the t-test result was non-significant $(\mathrm{t}=0.2799)$.

\section{INTERPRETATION OF RESULTS}

Overall, the results partially confirmed our expectations. As expected, we found H1 confirmed, suggesting that recall, and thus also acquisition of simple factual knowledge is better facilitated through gaming than transfer of complex knowledge. At the same time, we noted that not even for simple knowledge there was complete recall 


\section{Issues in Information Systems \\ Volume 14, Issue 2, pp.367-374, 2013}

(thus disconfirming H2a), despite the fact that subjects were able to complete the tasks which they were tested on. This suggests that subjects learn "how-to" knowledge related to the task, but not necessarily build up corresponding explanation skills. This is not entirely surprising, especially for complex knowledge, considering the following example. Subjects had to master a task scenario where their game character climbs one flight of stairs, then another, then another, until reaching the goal location. While subjects may be able to successfully complete this task, they may not create the awareness that they climbed exactly three (virtual) staircases. Similarly, they may also recall the visual cues to collect a certain object type (e.g., white flower), but when asked, may not recall that the color of the flower was white.

Overall, we expected higher performance for factual knowledge acquisition than the findings indicate. Nevertheless, we must realize the considerable difference between the ability to perform a task, virtual or real, and the ability to create awareness about it and to verbalize it.

Finally, we noted that $\mathrm{H} 3 \mathrm{a}$ was only confirmed at $\mathrm{p}=0.1$ (0.083), whereas $\mathrm{H} 3 \mathrm{~b}$ was rejected. Nevertheless, considering the difference in completion rates between the two subject groups $(93 \%$ for the chauffeured group and $65 \%$ for the self managed group), we infer that some level of initial supervision (chauffeuring) was vital to acquiring critical knowledge needed to overcome initial steep portions of the learning curve. We might expect that given a larger data set, the results could become significant, and simply note that even a well-developed game such as WoW at Level 1, can pose a considerable challenge to novice users. This is exemplified in the open ended comments at the end of the questionnaire where subjects share difficulties in navigation, and sense making of the 3D environment. As more organizations attempt to explore the benefits of game-based learning (serious games), one implication of our findings would be the need to include some form of initial supervision (chauffeuring), in order to flatten initially steep challenges and reduce the overall number of participants who "give up".

\section{LIMITATIONS}

Our study has numerous limitations, of course. One of the key shortcomings is the small sample size, especially given the high failure-to-complete rate. Unfortunately, the experimental task requires about an hour per subject, and chauffeuring is resource intensive. Nevertheless, enlarging the sample size is expected to generate more meaning, especially with respect to the impact of chauffeuring.

Another shortcoming is the choice of questions that subjects have to answer. We already generated a broad set of 14 questions in the post-experiment questionnaire, and chose questions we believed to be task-related (e.g., "how to do you approach the quest giver"). Nevertheless, we should not expect subjects to acquire knowledge such as the number of flight of flights of stairs their character has to climb, as subjects in the game will simply learn by-doing to climb until they reach the destination. Thus, poor questioning may bias outcomes. To overcome potential bias we asked many questions, and carefully reviewed the questions prior to the experiment, yet more, or more task (doing) related, questions may be needed.

A further important shortcoming is the lack of a control. We measured knowledge acquisition through gaming, but not through other means. We could have questioned subjects after the chauffeuring and measure their recall, or we could have given them a gaming guide, many of which are available, and let them study just the guide prior to answering questions. In that way, we could have created some benchmarks for knowledge transfer using passive gaming or simply based on reading. To note, Oz \& White [18] for instance reported different retention rates for different presentation formats ( $75 \%$ for seeing and hearing and doing, $40 \%$ for seeing and hearing, $20 \%$ for hearing).

\section{CONCLUSIONS}

In this experiment, we set out to examine knowledge acquisition within the context of gaming. Our results provide several interesting insights. First, we showed that people acquire and retain knowledge as they play games. It may be argued that "learning while playing" is rather intuitive, more of a function of human memory as opposed to special qualities of games. However, it is still valuable to empirically show that aside from the entertainment value derived from playing games, players are able to retain knowledge. This level of knowledge acquisition and recall varies with knowledge complexity. Not only are people able to succeed in complex quests, they also seem to recall 


\section{Issues in Information Systems \\ Volume 14, Issue 2, pp.367-374, 2013}

quest related knowledge at a level of about $80 \%$ for simple, factual knowledge, and 55\% for complex, conceptual and planning knowledge. Next, our results shows that while people play games they are able to acquire knowledge that falls within the tacit spectrum. This also demonstrates that gaming offers opportunity to practice skills that involve the ability to learn through experience, non-verbal cues and observing the effects of actions, without subjects always clearly realizing that they do. At a more granular level, we realize that an initial period of chauffeuring aids task completion but not explicit knowledge acquisition.

\section{ACKNOWLEDGMENT}

This research was supported in part by CityU Seed Grant No. 7003057.

\section{REFERENCES}

1. Bartle, R. (2003). Design of Virtual Worlds. New Riders Publishing.

2. Bates, J. (1992). Virtual reality, art, and entertainment. Presence: The Journal of Teleoperators and Virtual Environments, 1(1), 133-138.

3. Bell, M.W. (2008). Toward a Definition of "Virtual Worlds". Journal of Virtual World Research, 1(1).

4. Bloom, B.S., Engelhart, M.D., Furst, E.J., Hill, W.H., \& Krathwohl, D. R. (1956). Taxonomy of educational objectives: Handbook I: Cognitive domain. New York: David McKay, 19, 56.

5. Boulos, M.N.K., Hetherington, L., \& Wheeler, S. (2007). Second Life: an overview of the potential of 3D virtual worlds in medical and health education. Health Information \& Libraries Journal, 24(4), $233-245$.

6. Brown, J., \& Thomas, D. (2006, April). You Play World of Warcraft? You're Hired! Wired, p. Issue 14.04.

7. Charles, D., \& McAllister, M. (2004). Integrating ideas about invisible playgrounds from play theory into online educational digital games. Entertainment Computing, 598-601.

8. Gee, J.P. (2008). Learning Theory, Video Games and Popular Culture. In J. P. Gee, International Handbook on Children, Media, Culture (pp. 2005-2006). Sage Publishing.

9. Gutierrez, F., Coulter, R., Saland, L., Caudell, T. P., Goldsmith, T.E. \& Alverson, D.C. (2007). The effect of degree of immersion upon learning performance in virtual reality simulations for medical education. Medicine Meets Virtual Reality 15: In Vivo, in Vitro, in Silico: Designing the Next in Medicine, 125, 155-160.

10. Hirumi, M., \& Kebritchi, A. (2008). Examining the pedagogical foundations of modern educational computer games. Computers \& Education, 1729-1743.

11. Holland, W., Jenkins, H., \& Squire, K. (2003). Theory by design. (B. Perron, \& M. Wolf, Eds.) Video game theory, Routledge.

12. Ju, E., \& Wagner, C. (1997). Personal Computer Adventure Games : Their Structure, Principles and Applicability for Training. The Database for Advances in Information Systems, 28(2), 78-92.

13. Krogh, G. V., Ichijo, K., \& Nonaka, I. (2000). Enabling Konwledge Creation : How to Unlock the Mystery of Tacit Knowledge. Oxford: Oxford University press.

14. Livingston, D., \& Kemp, J. (2006). Massively Multi-Learner: Recent Advances in 3D Social Environments. Computing and Information Systems Journal, 10(2).

15. MacCrimmon, K.R., \& Taylor, R.N. (1976). Decision making and problem solving. Handbook of industrial and organizational psychology. Chicago: Rand-McNally.

16. Newell, A., \& Simon, H. A. (1972). Human problem solving. Englewood Cliffs, NJ: Prentice-Hall.

17. Nonaka, I., \& Takeuchi, H. (1995). The Knowledge-Creating Company: How Japanese Companies Create the Dynamics of Innovation. Oxford: Oxford University Press.

18. Oz, E., \& White, L. (1993). Multimedia for Better Training. Journal of Systems Management, May, 55-58.

19. Polanyi, M., \& Sen, A. (1966). The Tacit Dimension. Chicago: The University of Chicago Press.

20. Pounds, W.F. (1969). The Process of Problem Finding. Industrial Management Review, 11(1), 1-19.

21. Schultze, U., \& Orlikowski, W.J. (2010). Virtual Worlds: A Performative Perspective on Globally Distributed, Immersive Work. Information Systems Research, 21(4), 810 - 821.

22. Sheffield, B. (2005). What games have to teach us: An interview with James Paul Gee. Game Developer, 12(10), 4-9.

23. Smith. (2001). The role of tacit and explicit knowledge in the workplace. Journal of Knowledge Management, 5(4), 311-321. 


\section{Issues in Information Systems}

Volume 14, Issue 2, pp.367-374, 2013

24. Taylor, T. (2002). The social life of avatars : Presence and interaction in shared virtual environments. In T. Taylor, \& R. Schroeder (Ed.), Living digital : Embodiment in virtual worlds (pp. 40-62). New York : SpringerVerlag.

25. Wu, J., \& Liu, D. (2007). The effects of trust and enjoyment on intention to play online games. Journal of Electronic Commerce Research, 8(2), 128-140.

26. Wowpedia. (2013, March 8). Experience Point. Retrieved from Wowpedia: http://www.wowpedia.org/Experience_point

27. Yee, N. (2006). The Demographics, Motivations, and Derived Experiences of Users of Massively Multi-User Online Graphical Environments. Presence: Teleoperators and Virtual Environments, 309 -329.

28. Yee, N. (2007). Motivations of Play in Online Games. Journal of CyberPsychology and Behaviour, 9, $772-775$. 